\title{
Supplementary Information: \\ Dynamic Imaging of Multiple SERS Hotspots on Single Nanoparticles
}

Carlos Diego L. de Albuquerque ${ }^{1,3}$, Kallai M. Hokanson ${ }^{2}$, Sarah R. Thorud ${ }^{2}$, Regivaldo G. Sobral-Filho ${ }^{13}$ Nathan C. Lindquist ${ }^{2 *}$, Alexandre G. Brolo ${ }^{1,3^{*}}$

${ }^{1}$ Department of Chemistry, University of Victoria, Victoria, BC, V8P 5C2, Canada

${ }^{2}$ Department of Physics and Engineering, Bethel University, 3900 Bethel Drive, St Paul, MN, 55112 USA

${ }^{3}$ Centre for Advanced Materials and Related Technologies (CAMTEC), University of Victoria, Victoria, BC, V8W 2Y2, Canada

*n-lindquist@bethel.edu; agbrolo@uvic.ca 
Silver nanoshells $\left(\mathrm{SiO}_{2} @ \mathbf{A g}\right)$ synthesis and characterization. SERS Silica cores with a 90 $\mathrm{nm}$ diameter and a narrow size distribution were first obtained by adding Tetraethyl orthosilicate (TEOS) to a reverse microemulsion of Triton X-100 dispersed in $\mathrm{n}$ hexanol/cyclohexane mixture. The silica core was modified with (3Aminopropyl)trimethoxysilane (APTMS) and then decorated with 2-3 nm gold nanoparticles. The gold nanoparticles served as seeds for the growth of a $15 \mathrm{~nm}$ silver shell from a plating solution $\left(\mathrm{AgNO}_{3}\right)$. A very detailed description of the synthesis can be found elsewhere. ${ }^{1,2}$ The $\mathrm{SiO}_{2} @ \mathrm{Ag}$ were then coated with DTNB (5,5'-dithiobis-(2-nitrobenzoic acid)). The S-S bond do DTNB is expect to dissociate and the molecular fragments bind onto the metallic surface by the thiol terminal group. The thiol-modified nanoparticles were then immobilized on a glass slide. The slides containing $\mathrm{SiO}_{2} @ \mathrm{Ag}$ nanoshells were dried and characterized by electron microscopy and dark field hyperspectral imaging (Figure 1, main text).

Raman spectrometer. SERS spectra were collected using a Raman confocal microscope (Renishaw) equipped with a $633 \mathrm{~nm}(\mathrm{He}-\mathrm{Ne})$ laser excitation, a $50 \mathrm{x}$ objective, notch filters and an Andor camera EM-CCD detector for fast acquisitions. Two experimental condition was used as described: (1) a single Raman spectrum was collected (Figure 1f) laser power was around $\sim 100 \mu \mathrm{W}, 10 \mathrm{~s}$ of integration and 1 accumulation. This measurement was carried out with resolution of $<1 \mathrm{~cm}^{-1}$ and in the range of $1000-1600 \mathrm{~cm}^{-1}$. The raw spectrum was then preprocessed by following steps: (a) noise removed using Savitzky-Golay filter ${ }^{3}$ and (b) the baseline was corrected using the asymmetric least-square method ${ }^{4}$; (2) a speed test was carried out to estimate the time recording by the Andor camera. A silicon wafer was used as a reference material because of its well-known band in @ $520 \mathrm{~cm}^{-1}$. Using different time integration, the number of counts can be estimated by integrating the intensity (counts) in the range of $520 \pm 50$ $\mathrm{cm}^{-1}$. A plotting of the integrated counts versus time is shown in Figure S1. Extrapolating the linear fitting, the integration time, set as " $0 \mathrm{~s}$ " in the Renishaw software, can be found as $\sim 90$ ms. The standard deviations indicate the variation of 1000 spectra for each respective time integration. A good statistic is observed by the narrow standard deviations and good correlation coefficient $\left(r^{2}=0.999\right)$. 


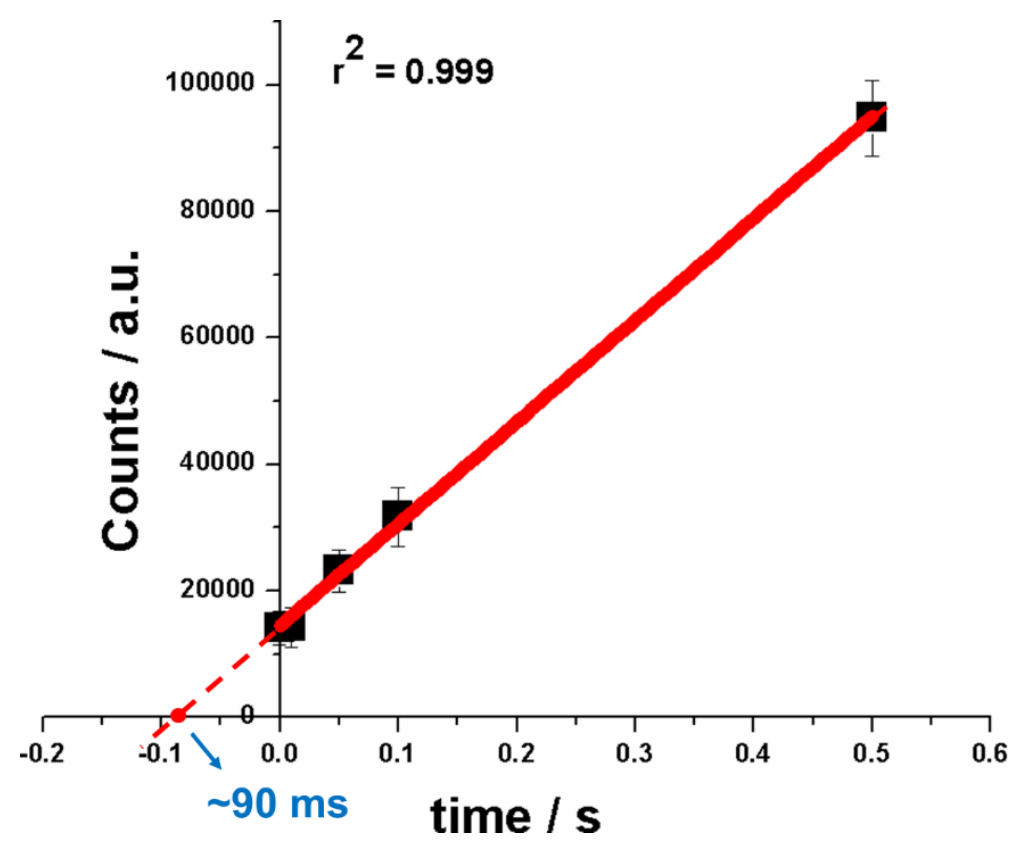

Figure S1. Time speed test using the Andor camera in the Raman spectrometer.

Confocal Laser Scanning Microscope (LSM). The measurements were performed using an inverted CLSM system (Zeiss LSM 880) with equipped with two He-Ne lasers for $543 \mathrm{~nm}$ and $633 \mathrm{~nm}$, an objective Plan-Apochromat 63x/1.4 NA Oil DIC 127, MBS: 543/633 nm and bandpass filters and two PMT detectors. The equipment is controlled by the software Zen black ${ }^{\circledR}$ where there is a wide number of options. Typically, the images were acquired with $3.9 \mathrm{x}$ $3.9 \mu \mathrm{m}^{2}$ (or $8 \times 8$ pixels), 16 bits of pixel depth, time dwelling of $1.23 \mu$ s per pixel and $200 \mathrm{fps}$. The laser powers at the sample were between $\sim 50$ to $\mu \mathrm{W} \sim 200 \mu \mathrm{W}$. The single pixel measurements were obtained in spot scan with dwelling time of $1.23 \mu$ s or 800,000 fps. All measurements were performed in room temperature at $\sim 20{ }^{\circ} \mathrm{C}$. The data were saved in .ome (bigTiff format) for helping to extract the frames in MATLAB® environment. All routines were written by the authors.

Polarization experiments. The polarization experiments were set up on a standard inverted microscope (Nikon) with custom laser illumination optics. The laser was a $660 \mathrm{~nm}$ solid state laser (Laser Quantum Gem 660). The polarization was controlled via a Liquid Crystal Variable Waveplate (LCVWP, Thorlabs LCC1223-A) that was able to provide a full wavelength of retardance at $660 \mathrm{~nm}$ controlable with an applied voltage. As described in the main text, the laser polarization excitation was alternated from $x$-polarized light to $y$-polarized light while the EMCCD camera (Andor iXon Ultra) recorded the SERS emission and SIF events. The LCVWP was set to alternate polarizations every 5 seconds and the camera recorded at a constant 15 frames per second, synchronized to the LCVWP via a trigger mechanism and function generator. The nanoshells were prepared as before and imaged with a $100 \mathrm{x}$ oil $(\mathrm{NA}=1.25)$ immersion objective. A long pass filter (Semrock) was used to block the laser line and image 
only the SERS emission. Image frames were processed either via a custom MATLAB® script as before or with an open-source super-resolution software package ${ }^{6}$ and the SERS emission centroids were localized.

Computer simulations. Finite element computer simulations were performed with the COMSOL $^{\mathrm{TM}}$ Multiphysics software with the Wave Optics module for simulating the field in and around a nanoshell particle. The bumpy particle geometry was first created in the free and opensource 3D design software Blender, using a procedural noise texture to generate a qualitatively similar surface to the TEM images shown in the main text. The geometry was then imported into COMSOL and the built-in material constants based on experimental values ${ }^{7}$ were used to simulate the silver nanoshell geometry. Perfectly Match Layers (PML) were used to absorb outgoing radiation and a focused Gaussian beam illuminated the particle at the various wavelengths and polarizations as described in the main text. The smallest mesh element was set to $2 \mathrm{~nm}$ and grew to $40 \mathrm{~nm}$ in the free-space regions. The intensity of the electric field was then plotted over the surface of the particle to visualize the plasmonic hotspots with the various illumination conditions. Temperature simulations are shown in Figure S2 below. These were done with an ambient environment temperature of $20^{\circ} \mathrm{C}$ and an illumination power of $100 \mu \mathrm{W}$ focused on the particle. Thermal material constants were taken from published values ${ }^{8,9}$. The high temperature increase on resonance is partly due to the surrounding environment being air.
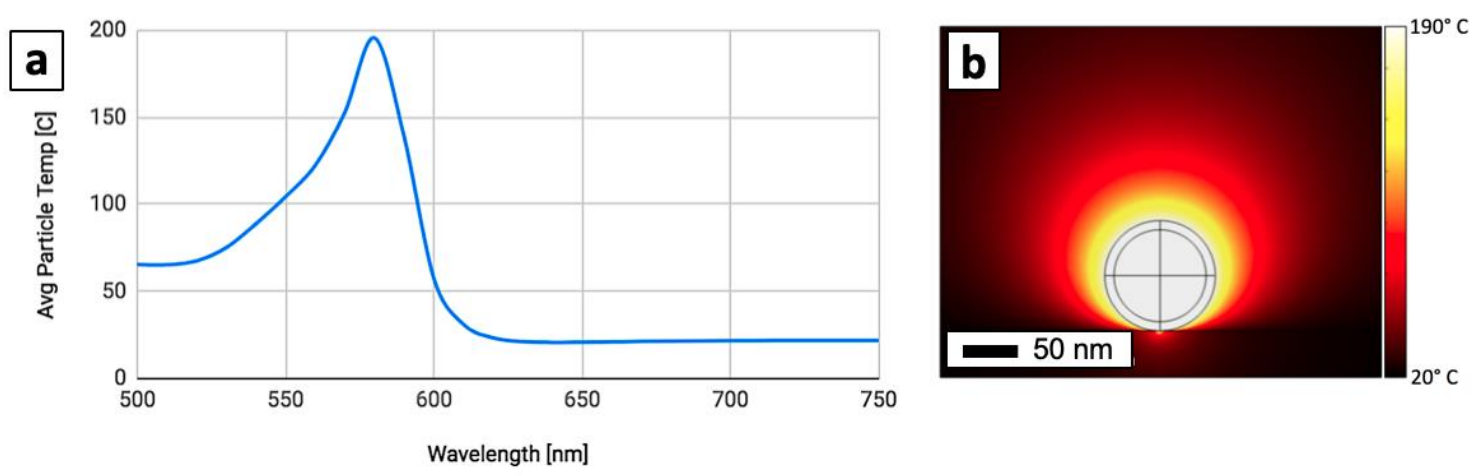

Figure S2. Temperature simulations. (a) The average temperature of a spherical nanoshell particle as a function of wavelength. The peak occurs around $\sim 580 \mathrm{~nm}$ with a maximum temperature of $\sim 190^{\circ} \mathrm{C}$, corresponding to a local temperature increase of $\sim 170^{\circ} \mathrm{C}$ from ambient. The green laser at $543 \mathrm{~nm}$ heats to $\sim 100^{\circ} \mathrm{C}$ whereas due to the LSPR of the nanoshell particle, the red laser will heat less. (b) Heat map of the temperature distribution in and around the particle on resonance. 

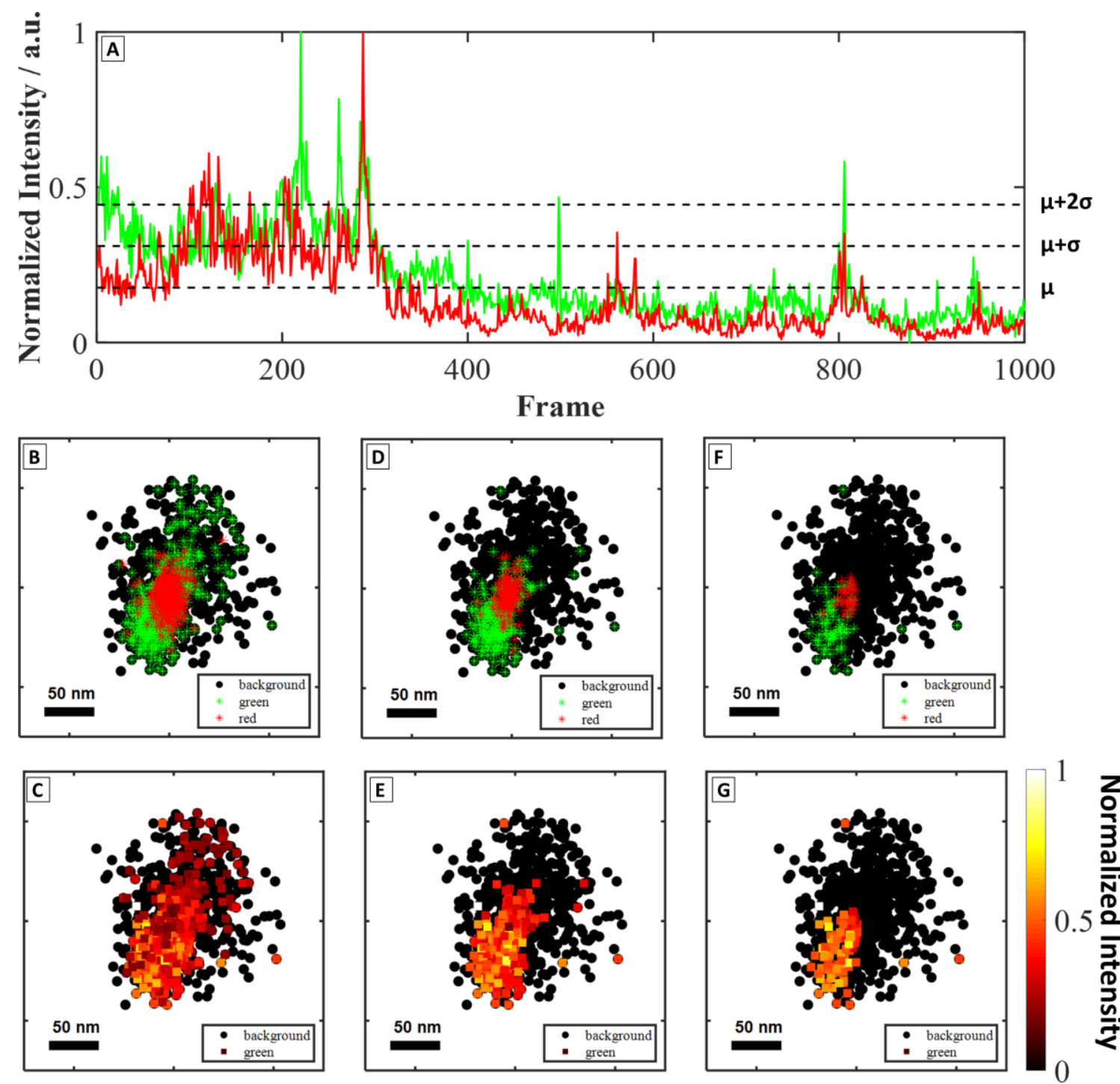

Figure S3. SERS intensity fluctuations (SIFs) for a single nanoparticle. (A) Simultaneous SERS trajectories for the green $(543 \mathrm{~nm})$ and red $(633 \mathrm{~nm})$ lasers. The centroid of the nanoparticle is set at coordinates $(0,0)$. Plot showing the SIF colocalization with different lasers (middle, B, D, F) and intensity distributions (bottom, C, E, G) with different thresholds. For (B) and (C) the threshold is the mean $\mu$. For (D) and (E) it is $\mu+\sigma$ where $\sigma$ is the standard deviation of the signal. For $(F)$ and $(G)$ the threshold is $\mu+2 \sigma$. These plots show that different hotspots are accessible with different lasers (i.e. red and green are from different regions of the particle) and they also present different strengths. The largest fluctuations are well distinguished from the background, i.e. the localizations from the entire trajectory. Differently than the Figure 4C in main text, here the red laser was located more in the center of the particle than on the edge, whereas the green SERS is coming from the bottom of the particle. This further emphasizes that the local nanostructuring within the nanoparticle plays an important role over the larger LSPR.

\section{References}

1. Brito-Silva, A. M.; Sobral-Filho, R. G.; Barbosa-Silva, R.; de Araújo, C. B.; Galembeck, A.; Brolo, A. G., Improved Synthesis of Gold and Silver Nanoshells. Langmuir 2013, 29 (13), 43664372.

2. $\quad$ Sobral-Filho, R. G.; Brito-Silva, A. M.; Isabelle, M.; Jirasek, A.; Lum, J. J.; Brolo, A. G., Plasmonic labeling of subcellular compartments in cancer cells: multiplexing with fine-tuned gold and silver nanoshells. Chem. Sci. 2017, 8 (4), 3038-3046. 
3. Savitzky, A.; Golay, M. J. E., Smoothing and Differentiation of Data by Simplified Least Squares Procedures. Analytical chemistry 1964, 36 (8), 1627-1639.

4. $\quad$ Eilers, P. H. C., Parametric Time Warping. Analytical chemistry 2004, 76 (2), 404-411.

5. Temple, P. A.; Hathaway, C. E., Multiphonon Raman Spectrum of Silicon. Physical

Review B 1973, 7 (8), 3685-3697.

6. Wolter, S.; Löschberger, A.; Holm, T.; Aufmkolk, S.; Dabauvalle, M.-C.; van de Linde, S.; Sauer, M., rapidSTORM: accurate, fast open-source software for localization microscopy.

Nature Methods 2012, 9, 1040.

7. Johnson, P. B.; Christy, R. W., Optical Constants of the Noble Metals. Physical Review B 1972, 6 (12), 4370-4379.

8. Young, H. D.; Sears, F. W., University physics. 8th ed. / ed.; Addison-Wesley Pub. Co.: Reading, Mass., 1992.

9. Tipler, P. A., Physics for Scientists and Engineers: Regular Version, Ch. 1-35 and 39. W.

H. Freeman: 1999. 